5. L. K. Hua, $A$ note on the total matrix ring over a non-commutative field, Annales de la Société Polonaise de Mathématique vol. 25 (1952) pp. 188-198.

6. N. Jacobson and C. E. Rickart, Jordan homomorphisms of rings, Trans. Amer. Math. Soc. vol. 69 (1950) pp. 479-502.

7. F. Kasch, Invariante Untermoduln des Endomorphismenrings eines Vektorraums, Archiv der Mathematik vol. 4 (1953) pp. 182-190.

8. - Über den Automorphismenring einfacher Algebren, Archiv der Mathematik vol. 6 (1955) pp. 59-65.

NORTHWESTERN UNIVERSITY

\title{
CANCELLATION IN DIRECT SUMS OF GROUPS
}

\section{ELBERT A. WALKER ${ }^{1}$}

1. Introduction. The purpose of this paper is to consider the following question for groups. If $F \oplus G=F^{\prime} \oplus H$ and $F \cong F^{\prime}$, when is $G \cong H$ ? It is easy to see that for $G$ to be isomorphic to $H$ some additional hypothesis must be given. For example let $C_{i}, i=1,2,3, \ldots$ be cyclic of order two. Let $F=C_{2} \oplus C_{3} \oplus C_{4} \oplus \cdots$, let $G=C_{1}$, let $F^{\prime}=C_{1} \oplus C_{3} \oplus C_{5} \oplus \cdots$, and let $H=C_{2} \oplus C_{4} \oplus C_{6} \oplus \cdots$. Clearly $F \oplus G=F^{\prime} \oplus H$ and $F \cong F^{\prime}$, yet $G$ not $\cong H$. One hypothesis that is suggested by this example is to require that $F$ be finitely generated. In fact, in Kaplansky's book, Infinite Abelian groups, p. 13, the author asks the following question for Abelian groups, called Test Problem III. If $F \oplus G=F^{\prime} \oplus H, F \cong F^{\prime}$, and $F$ is finitely generated, is $G \cong H$ ? The main results of this paper are theorems from which Test Problem III follows as a corollary.

2. Notations. The additive notation for groups will be used. The symbol $F \oplus G$ will denote the direct sum of the two groups $F$ and $G$. The commutator subgroup of a group $G$ will be denoted by $Q(G)$. The symbol $\{S\}$ will denote the group generated by the set $S$ of elements. The order of an element $g$ of $G$ will be written $o(g)$, and $o(S)$ will denote the number of elements in the set $S$. The infinite cyclic group will be represented by $C$, and the cyclic group of order $n$ by $C(n)$. The symbol $Z(G)$ will denote the center of the group $G$.

3. Definitions. Suppose $F \oplus G=F^{\prime} \oplus H$. The set of $F$ components of the elements of $F^{\prime}$ will be denoted by $F_{1}$, and the set of $F$ com-

Presented to the Society, September 2, 1955 under the title The solution of Kaplansky's test problem III; received by the editors October 4, 1955.

1 University Fellow, University of Kansas. 
ponents of the elements of $H$ will be denoted by $F_{2}$. The sets $G_{1}, G_{2}$, $F_{1}^{\prime}, F_{2}^{\prime}, H_{1}$, and $H_{2}$ are defined analogously. All these sets are normal subgroups of $F \oplus G$.

4. Theorem. If $F \oplus G=F^{\prime} \oplus H$ then $F+H=F \oplus G_{2}=F_{1}^{\prime} \oplus H$.

Proof. It is easy to see that $F+H=F \oplus((F+H) \cap G)$. Let $g$ be in $(F+H) \cap G$. Then $g=f+h$ with $f$ in $F$ and $h$ in $H$. But $h=f_{2}+g_{2}$ with $f_{2}$ in $F_{2}$ and $g_{2}$ in $G_{2}$. Hence $g=f+f_{2}+g_{2}$. Since any element can be written in exactly one way as the sum of an element of $F$ and one of $G$, it follows that $f+f_{2}=0$ and $g=g_{2}$. Thus $(F+H) \cap G \subseteq G_{2}$. Let $g_{2}$ be any element of $G_{2}$. Then $\left(f+g_{2}\right)$ is in $H$ for some $f$ in $F$. Then $-f+\left(f+g_{2}\right)=g_{2}$ is in $G_{2}$ so that $G_{2} \subseteq F+H$. Hence $G_{2} \subseteq(F+H) \cap G$, and we get $F+H=F \oplus G_{2}$. Similarly $F+H=F_{1}^{\prime} \oplus H$.

5. Theorem. If $F \oplus G=F^{\prime} \oplus H$, then $F /(F \cap H) \cong F_{1}^{\prime}$, and $H /(F \cap H)$ $\cong G_{2}$.

Proof. Project $F$ onto its $F^{\prime}$ components. The range of this projection is $F_{1}^{\prime}$ and the kernel is $F \cap H$. Hence $F /(F \cap H) \cong F_{1}^{\prime}$. Similarly $H /(F \cap H) \cong G_{2}$.

6. Theorem. If $F \oplus G=F^{\prime} \oplus H, F \cong F^{\prime}$, and $F$ is finite then $G \cong H$.

Proof. By $\S 4, F+H=F \oplus G_{2}=F_{1}^{\prime} \oplus H$. If $F \cap H=0$, then $F \oplus H$ exists and $F \oplus H=F_{1}^{\prime} \oplus H$, so that $F \cong F_{1}^{\prime}$. Since $F$ is finite and $F \cong F^{\prime}$, it follows that $F_{1}^{\prime}=F^{\prime}$ and $F \oplus H=F^{\prime} \oplus H=F \oplus G$. Hence $G \cong H$. If $F \cap H \neq 0$, we proceed by induction on $o(F)=o\left(F^{\prime}\right)$. If $o(F)=1$ the theorem is obvious. Since $F \oplus G=F^{\prime} \oplus H$ we get that $F /(F \cap H) \oplus G \cong F^{\prime} \oplus H /(F \cap H) . F^{\prime} \cong F$ and $H /(F \cap H) \cong G_{2}$ by $\S 5$ implies that $F /(F \cap H) \oplus G \cong F \oplus G_{2}=F_{1}^{\prime} \oplus H$. By $5, F /(F \cap H) \cong F_{1}^{\prime}$ and since $F \cap H \neq 0$, it follows that $o\left(F_{1}^{\prime}\right)<o(F)$. Hence our induction hypothesis implies that $G \cong H .^{2}$

7. Theorem. If $F \oplus G=F^{\prime} \oplus H, F \cong F^{\prime} \cong C$, and $G$ is Abelian, then $G \cong H$.

Proof. Let $P$ be the projection $\left(f^{\prime}+h\right) P=f^{\prime}$, where $f^{\prime}$ is in $F^{\prime}$ and $h$ is in $H$. Then $P$ is a homomorphism of $G$ into $F^{\prime}$ with kernel $G \cap H$. If $G P=0$, then $G \subseteq H$ and since $G$ is a direct summand of a group containing $H$, we get $H=G \oplus H^{\prime}$ for some group $H^{\prime}$. Hence $F \oplus G=F^{\prime} \oplus G \oplus H^{\prime}$ and $F \cong F^{\prime} \oplus H^{\prime}$. Since $F \cong F^{\prime} \cong C$, it follows that $H^{\prime}=0$ and $G=H$. If $G P \neq 0$ then $G P$ is infinite cyclic, so that $G /(G \cap H) \cong C$, from which follows that $G \cong(G \cap H) \oplus C$ since $G$ is

${ }^{2}$ Bjarni Jonsson has informed me that this result follows from Theorem 3.11 in the book Direct Decomposition of Finite Algebraic Systems, by Jonsson and Tarski. 
Abelian. The group $H$ is also Abelian so by symmetry we may assume $H \cong(H \cap G) \oplus C$. Hence $G \cong H$.

8. Corollary (Kaplansky's Test Problem III). If $F \oplus G=F^{\prime} \oplus H$, $F \cong F^{\prime}$ and $F$ is finitely generated, and $F$ and $G$ are Abelian, then $G \cong H$.

Proof. Since $F$ is Abelian and finitely generated it is the direct sum of a finite group and a finite number of infinite cyclic groups. Clearly it suffices to prove $G \cong H$ for the two cases $F$ finite and $F$ infinite cyclic. But these two cases are proved in $\$ \$ 6$ and 7 . Hence $\$ 8$ follows.

9. Corollary. The decomposition of a finitely generated Abelian group into indecomposable summands is unique up to isomorphism.

Proof. Let $F_{1} \oplus F_{2} \oplus \cdots \oplus F_{m}=F_{1}^{\prime} \oplus F_{2}^{\prime} \oplus \cdots \oplus F_{n}^{\prime}$ be two decompositions of a finitely generated Abelian group into indecomposable summands. Then each $F_{i}$ and $F_{k}^{\prime}$ is cyclic of prime power order or infinite cyclic. We induct on $m$. If $m=1$, then $\$ 9$ follows because $F_{i}$ and $F_{k}^{\prime}$ are indecomposable. Suppose the left side has an infinite cyclic summand. Then so does the right. Let for convenience these two summands be $F_{1}$ and $F_{1}^{\prime}$. Then by $\S 7, F_{2} \oplus F_{3} \oplus \cdots \oplus F_{m}$ $\cong F_{2}^{\prime} \oplus F_{3}^{\prime} \oplus \cdots \oplus F_{n}^{\prime}$ and by the induction hypothesis $\S 9$ follows.

Now we may assume all the summands on the left side are finite. Let $F_{i}$ be a summand of maximal prime power order, say $p^{r}$. Hence $F_{i}$ has an element $f_{i}$ of order $p^{r}$. Furthermore $f_{i}=f_{1}^{\prime}+f_{2}^{\prime}+\cdots+f_{n}^{\prime}$ and some $f_{j}^{\prime}$ is of order $p^{r}$. If $o\left(f_{j}^{\prime}\right)<o\left(F_{j}^{\prime}\right)$ then the right side has an element of order $p^{s}$ with $r<s$ and the left does not. Hence $o\left(F_{j}^{\prime}\right)$ $=o\left(F_{i}\right)$ and by $\S 6$ and the induction hypothesis we are done.

10. Theorem. If $F \oplus G=F^{\prime} \oplus H$, then the following are true:

(a) $Q\left(F_{1}\right) \subseteq F \cap F^{\prime}$.

(b) $Q\left(F_{2}\right) \subseteq F \cap H$.

(c) $Q(F)=Q\left(F_{1}\right) \oplus Q\left(F_{2}\right)$.

(d) $Q(F)=Q\left(F_{1}^{\prime}\right) \oplus Q\left(H_{1}\right)$.

Proof. The proof is straightforward and routine, so is omitted.

11. Theorem. If $F \oplus G=F^{\prime} \oplus H, F \cong F^{\prime} \cong C$, then the following are true:

(a) $Q(G)=Q(H)$.

(b) $G / Q(G) \cong H / Q(H)$.

(c) The group $G$ is isomorphic to a normal subgroup of $H$ of finite index in $H$. In fact $G \cong H$ or $G \cong H_{2}$.

(d) The group $H$ is isomorphic to a normal subgroup of $G$ of finite index in $G$. In fact $H \cong G$ or $H \cong G_{2}$. 
(e) If $Z(G)$ is torsion then $G \cong H$. (In particular, if $G$ is finite or if $Z(G)=0$, then $G \cong H$.)

(f) If $Q(G)=G$ then $G \cong H$.

Proof. (a) Using $\$ 10$ applied to $G$ and $H$ and the fact that $F$ and $F^{\prime}$ are Abelian, we get that $Q(G)=Q\left(G_{2}\right)$ and $Q(H)=Q\left(H_{2}\right)$. But $Q(G)=Q\left(F_{2}^{\prime}\right) \oplus Q\left(H_{2}\right)=Q\left(H_{2}\right)$ implies $Q(G)=Q(H)$.

(b) Since $Q(G)=Q(H)$ we get $F \oplus G / Q(G) \cong F^{\prime} \oplus H / Q(H)$. The groups $G / Q(G)$ and $H / Q(H)$ are Abelian. By $\S 7, G / Q(G) \cong H / Q(H)$.

(c)-(d) Let $F=\{f\}$ and $F^{\prime}=\{n f+g\}$. Then $f=(m n f+m g)$ $+((1-m n) f-m g)$, with $m n f+m g$ in $F^{\prime}$ and $((1-m n) f-m g)$ in $H$. If $n=0$ then $F^{\prime} \subseteq G$. In that case $G=F^{\prime} \oplus G^{\prime}$, for some $G^{\prime}$. Thus $F \oplus F^{\prime} \oplus G^{\prime}=F^{\prime} \oplus H$ and so $H \cong F \oplus G^{\prime} \cong F^{\prime} \oplus G^{\prime}$. Hence $G \cong H$. If $m=0$ then $F \subseteq H$, and similarly we get $G \cong H$. If $m n=1$ then $F \cap H=0$ and the projection of $H$ onto $G_{2}$ is an isomorphism. Since $\left\{G_{2}, g\right\}=G$, and $m g$ is in $G_{2}$, it follows that the index of $G_{2}$ in $G$ is finite. If $m n \neq 1$ and $m n \neq 0$, then $F \cap H=0$ because $o(f)$ is infinite and so the $F^{\prime}$ component of no nonzero element of $F$ is 0 . As in the previous case, $H \cong G_{2}$. By symmetry we may likewise show that $G \cong H$ or $G \cong H_{2}$. Thus (c) and (d) are proved.

(e) In proving (c) and (d) it was shown that either $G \cong H$ or $F \cap H$ $=0$. If $F \cap H=0$ then since $((1-m n) f-m g)$ is the $H$ component of $f$, it follows that $o(g)$ is infinite. But $F^{\prime}=\{n f+g\}$ implies that $g$ is in $Z(G)$. Thus if $Z(G)$ is torsion, $F \cap H \neq 0$ and $G \cong H$.

(f) If $Q(G)=G$, then by (b), $Q(H)=H$. Using (a), we get $G=H$.

12. Remark. Professor W. R. Scott constructed the interesting example given in the next theorem.

13. Theorem. There exist groups $G$ and $H$ such that $G$ not $\cong H$, yet $F \oplus G=F^{\prime} \oplus H$ with $F \cong F^{\prime} \cong C$.

Proof. Let $G \cap H=\{x\}$ with $11 x=0$. Let $G$ be the extension of $G \cap H$ by the automorphism $A$ such that $x A=2 x$. That is, $G=\{x, y\}$ where $-y+x+y=2 x$ and $G /(G \cap H) \cong\{y\} \cong C$. Let $H$ be the extension of $G \cap H$ by the automorphism $A$ such that $x A=8 x$. That is, $H=\{x, z\}$ where $-z+x+z=8 x$ and $H /(G \cap H) \cong\{z\} \cong C$. Now let $F=\{7 y+z\}, F^{\prime}=\{y+3 z\}$, and $z+y=y+z$. It is straightforward to prove that the groups just defined have the desired properties.

14. Theorem. If $F \oplus G=F^{\prime} \oplus H, F \cong F^{\prime}, F$ is indecomposable, and $Q(F)=F$, then $G \cong H$.

Proof. By $\S 10(\mathrm{c}), Q(F)=Q\left(F_{1}\right) \oplus Q\left(F_{2}\right)$. Since $F$ is indecomposable, $Q(F)=Q\left(F_{1}\right)$ and $Q\left(F_{2}\right)=0$, or $Q(F)=Q\left(F_{2}\right)$ and $Q\left(F_{1}\right)=0$. In the first 
case $F \subseteq F^{\prime}$ and by $\S 10(\mathrm{~d}), F=Q(F)=Q\left(F_{1}^{\prime}\right)$. Since $F \cong F^{\prime}$ is indecomposable and $F^{\prime}=Q\left(F^{\prime}\right)=Q\left(F_{1}^{\prime}\right) \oplus Q\left(F_{2}^{\prime}\right)$ it follows that $F=F^{\prime}$ and that $G \cong H$. Similarly if $Q(F)=Q\left(F_{2}\right)$ and $Q\left(F_{1}\right)=0$ then $G \cong H$.

15. Corollary. If $F \oplus G=F^{\prime} \oplus H, F \cong F^{\prime}$ and $F$ is simple, then $G \cong H$.

Proof. If $F$ is non-Abelian, $\$ 15$ follows from $\$ 14$. If $F$ is Abelian $\S 15$ follows from $\$ 6$.

16. Concluding remarks. The theorems given in $\$ \$ 4,5$, and 6 can be generalized to operator groups. In $\$ 6$, instead of requiring in the operator group case that $F$ be finite, one requires that $F$ have only a finite number of admissible subgroups. Kaplansky's Test Problem III may be extended to modules over principal ideal rings, and if $F$ is torsion, to modules over Dedekind rings. The proof given in $\$ 9$ of the uniqueness of the decomposition of finitely generated Abelian groups is, in this writer's opinion, a much better proof than has existed heretofore.

UNIVERSITY OF KANSAS 